\title{
Symplectic Quantization I: Dynamics of Quantum Fluctuations in a Relativistic Field Theory
}

\author{
Giacomo Gradenigo ${ }^{1}$ (D) $\cdot$ Roberto Livi $^{2,3,4}$
}

Received: 15 April 2021 / Accepted: 15 May 2021 / Published online: 26 May 2021

(c) The Author(s) 2021

\begin{abstract}
We propose here a new symplectic quantization scheme, where quantum fluctuations of a scalar field theory stem from two main assumptions: relativistic invariance and equiprobability of the field configurations with identical value of the action. In this approach the fictitious time of stochastic quantization becomes a genuine additional time variable, with respect to the coordinate time of relativity. This intrinsic time is associated to a symplectic evolution in the action space, which allows one to investigate not only asymptotic, i.e. equilibrium, properties of the theory, but also its non-equilibrium transient evolution. In this paper, which is the first one in a series of two, we introduce a formalism which will be applied to general relativity in its companion work (Gradenigo, Symplectic quantization II: dynamics of space-time quantum fluctuations and thecosmological constant, 2021).
\end{abstract}

\section{Introduction}

Beyond the notion that there is no absolute time flowing identically for observers in different reference frames of the universe, special relativity has challenged the classical concept of elementary particle, through the equivalence between mass and energy. In fact, fields rather than particles are the fundamental objects, which are consistent with a genuine relativistic description. They are represented as

Giacomo Gradenigo

giacomo.gradenigo@gssi.it

Roberto Livi

livi@fi.infn.it

1 Gran Sasso Science Institute, Viale F. Crispi 7, 67100 L'Aquila, Italy

2 Dipartimento di Fisica e Astronomia and CSDC, Università di Firenze, via G. Sansone 1, 50019 Sesto Fiorentino, Italy

3 Istituto Nazionale di Fisica Nucleare, Sezione di Firenze, via G. Sansone 1, 50019 Sesto Fiorentino, Italy

4 Consiglio Nazionale delle Ricerche, Istituto dei Sistemi Complessi, via Madonna del Piano 10, 50019 Sesto Fiorentino, Italy 
non-smooth functions, characterized by infinitely-many degrees of freedom, which describe the distribution of matter and/or radiation in the space-time continuum. In this manuscript we discuss a new pathway for inferring the origin of quantum fluctuations in a relativistic field theory, making use of concepts borrowed from statistical mechanics. More precisely, we are going to argue that quantum fluctuations in a relativistic field theory can be derived from a statistical description relying upon a microcanonical formulation, where the conserved quantity is the action, which is the only relativistically invariant scalar quantity. Due to some additional "kinetic" degrees of freedom of the quantum field, which is our care to introduce and motivate in the following discussion, one is able te set up a formalism where the sequence of quantum fluctuations experienced by a relativistic quantum field stems from a symplectic dynamics parametrized by an additional time variable, rather than from the stochastic process of the Parisi-Wu formalism [2].

There are multiple reasons to support this approach. The microcanonical generating function, i.e. the entropy functional, provides the same predictions of the standard path-integral formulation (e.g. see [3-5]), by which one can estimate the equilibrium correlation functions of the field at different points in space-time, provided one can establish the formal equivalence between a microcanonical formulation and a canonical one. Notice that this "ensemble equivalence" is not granted a priori for any relativistic field theory, in analogy with what happens in models of classical statistical mechanics: for instance, violations of this equivalence may emerge as a consequence of localization/condensation phenomena, induced by some peculiar form or symmetries of the interaction potential [6]. On the other hand, a microcanonical formulation which keeps the total action constant has no conceptual and physical contraindications for being adopted, independently of the interaction potential, as long as we make reference to an isolated system, as, for instance, it is the case of a closed universe. Moreover, in the natural Minkowski metric the action functional is not positive definite and this prevents the possibility of establishing a formal correspondence between the microcanonical entropy and the corresponding canonical potential (the equivalent of the free-energy) by a Laplace-transform. Conversely, one can always define a Fourier-transform of the entropy functional, which straightforwardly yields the standard Feynman path-integral formulation, once the Fourier dual variable of the action is attributed the value $\hbar^{-1}$. It is for this necessity to use the Fourier integral transform rather than the Laplace one that, at variance with ordinary statistical mechanics, the building blocks of quantum field theory are probability amplitudes rather than probabilities.

The main conceptual implication of our microcanonical approach is that the action can be used as the generator of a symplectic dynamics, associated to an intrinsic time $\tau$, replacing the fictitious time introduced by stochastic quantization $[2,4]$. This intrinsic time governs the evolution of quantum fluctuations and it has to be distinguished from the fourth coordinate of the relativistic space-time.

The main advantage of introducing symplectic dynamics in intrinsic time is that this provides a consistent (Lorentz invariant) and natural framework to study the non-equilibrium relaxational dynamics for a relativistic field quantum fluctuations. Symplectic quantization is free from the arbitrariness usually hidden in the choice of a noise term, typical of any Langevin-type dynamics, an arbitrariness which is particularly disturbing 
when noise is used to model the quantum fluctuations of relativistic fields. Moreover, symplectic quantization allows to define a functional approach to quantum field theory [see, e.g., Eqs. (22) and (28)] which is well defined and physically motivated irrespectively to the appearance of infinities from the path integral.

In what follows we provide a mathematical illustration of the program just outlined. In Sect. 2 we go through the main steps of the standard stochastic quantization, making reference, for the sake of simplicity, to the basic example of a scalar field, subject to a self-interaction potential. In Sect. 3 we discuss how a symplectic dynamical formalism can be introduced for the Euclidean formulation of the Lagrangian density and show that one can pass to the more natural formulation in terms of the action, expressed in its natural Minkowski metric. Comments and perspectives are contained in Sect. 4.

\section{Stochastic Quantization}

Since our method is inspired by the Parisi-Wu [2] stochastic quantization approach, we first summarize it by exploiting the elementary example of a neutral relativistic invariant scalar field subject to a self-interaction potential $V(\phi)$. The Lagrangian density of this model is:

$$
\begin{aligned}
\mathcal{L}\left(\phi, \partial_{\mu} \phi\right) & =\frac{1}{2} \partial^{\mu} \phi \partial_{\mu} \phi-V(\phi) \\
& =\frac{1}{2}\left(\frac{\partial \phi}{\partial x^{0}}\right)^{2}-\frac{1}{2} \sum_{i=1}^{3}\left(\frac{\partial \phi}{\partial x^{i}}\right)^{2}-V(\phi),
\end{aligned}
$$

where $x_{0}=c t$ denotes the coordinate time. The textbook case is the nonlinear Klein-Gordon model, where $V(\phi)=\frac{1}{2} m \phi^{2}+\frac{\lambda}{4 !} \phi^{4}$. The integral over the space-time continuum of the Lagrangian density in Eq. (1) is a relativistic invariant, the action

$$
S[\phi]=\int d x^{0} d \mathbf{x} \mathcal{L}\left(\phi, \partial_{\mu} \phi\right) .
$$

Due to the Maupertuis least action principle, the classical configurations of the field are those which extremize the action:

$$
\frac{\delta S}{\delta \phi\left(x^{0}, \mathbf{x}\right)}=0 .
$$

According to the path-integral formalism, the generating function for all the correlations among quantum fluctuations of the theory is the partition function

$$
\mathcal{Z}=\int \mathcal{D} \phi \exp \left\{\frac{i}{\hbar} S[\phi]\right\} .
$$

If one then considers the coordinate time $x^{0}$ as a variable in the complex plane and performs a Wick rotation, introducing the new variable $\tilde{x}^{0}=e^{i \pi / 2} x^{0}=i x^{0}$ the Lagrangian density changes to 


$$
\begin{aligned}
\mathcal{L}_{E} & =-\left[\frac{1}{2}\left(\frac{\partial \phi}{\partial \tilde{x}^{0}}\right)^{2}+\frac{1}{2} \sum_{\mu=1}^{3}\left(\frac{\partial \phi}{\partial x^{i}}\right)^{2}+V(\phi)\right] \\
& =-\mathcal{L}^{(+)}\left(\phi, \partial_{\mu} \phi\right),
\end{aligned}
$$

where we have denoted the term between square brackets on the right hand side of Eq. (5) as $\mathcal{L}^{(+)}\left(\phi, \partial_{\mu} \phi\right)$, to indicate that it corresponds to a Lagrangian with the wrong sign of the potential energy. In fact, if we really regard $\tilde{x}^{0}$ just as an additional spatial coordinate, $\mathcal{L}^{(+)}\left(\phi, \partial_{\mu} \phi\right)$ corresponds to a Hamiltonian density without the kinetic energy, since all terms with derivatives formally belong to a potential energy term. After Wick's rotation the action reads

$$
S[\phi]=i \int d \tilde{x}^{0} d \mathbf{x} \mathcal{L}^{(+)}\left(\phi, \partial_{\mu} \phi\right),
$$

and the corresponding partition function has the form

$$
\begin{aligned}
\mathcal{Z} & =\int \mathcal{D} \phi \exp \left\{-\frac{1}{\hbar} \int d^{4} x \mathcal{L}^{(+)}\left(\phi, \partial_{\mu} \phi\right)\right\} \\
& =\int \mathcal{D} \phi \exp \left\{-\frac{1}{\hbar} S^{(+)}[\phi]\right\} .
\end{aligned}
$$

From Eq. (7) it is clear the formal analogy with a statistical mechanics model where $S^{(+)}[\phi]$ plays the role of the Hamiltonian and $\hbar$ the one of temperature. The multipoint correlation functions read:

$$
\langle\phi(x) \phi(y) \ldots \phi(z)\rangle=\frac{1}{\mathcal{Z}} \int \mathcal{D} \phi e^{-(1 / \hbar) S^{(+)}[\phi]} \phi(x) \phi(y) \ldots \phi(z)
$$

The main argument for establishing a relation between the Euclidean path-integral of Eqs. (7) and (8) and stochastic quantization [2] is very simple (e.g., see $[2,4,7])$. In order to sample the configurations of the field $\phi(x)$ with probability $\exp \left\{-(1 / \hbar) S^{(+)}[\phi]\right\}$ one usually sets up a Monte Carlo simulation exploiting the above Boltzmann weight to build a Metropolis update rule. On the other hand, this fictitious stochastic process is essentially equivalent to define a stochastic dynamics based on a Langevin equation, where it appears a new time variable $\tau$, which in [2] was attributed the interpretation of a fictitious time. Since $\tau$ parametrizes the "internal" dynamics of quantum fluctuations independently to the choice of the reference frame, here we prefer to call it intrinsic time. Stochastic quantization amounts to introduce the following Langevin dynamics

$$
\frac{\partial}{\partial \tau} \phi(x, \tau)=-\frac{\delta S^{(+)}}{\delta \phi(x, \tau)}+\eta(x, \tau),
$$

where we have made explicit the dependence of the field also on $\tau$ and $\eta(x, \tau)$ is a Gaussian random field, defined by its mean value and by its correlation function: 


$$
\begin{aligned}
\langle\eta(x, \tau)\rangle & =0 \\
\left\langle\eta(x, \tau) \eta\left(y, \tau^{\prime}\right)\right\rangle & =2 \hbar \delta^{(4)}(x-y) \delta\left(\tau-\tau^{\prime}\right)
\end{aligned}
$$

The stochastic equation (9) was proposed in [2] as an alternative approach for sampling in the limit $\tau \rightarrow \infty$ the relativistic quantum fields according to their Euclidean equilibrium probability measure. In particular the main motivation of the stochastic quantization approach was that, when dealing with gauge fields, it does not require any gauge-fixing procedure [2]. This is why attempts in the direction of quantizing also gravity with this approach [8] were made shortly after the original proposal of the method for non-abelian gauge theories [2]. The generalization of the symplectic quantization method presented in this paper to gravity will be the main topic of [1]. The most important prescription of stochastic quantization is that multipoint correlation functions such as $\langle\phi(x) \phi(y) \ldots \phi(z)\rangle$ can be obtained by averaging over the stochastic trajectories generated by the Langevin dynamics (9) in the limit of infinite time, $\tau \rightarrow \infty$ : in formulae

$$
\lim _{\tau \rightarrow \infty}\langle\phi(x, \tau) \phi(y, \tau) \ldots \phi(z, \tau)\rangle_{\mathcal{D}}=\langle\phi(x) \phi(y) \ldots \phi(z)\rangle,
$$

where the subscript $\mathcal{D}$ denotes the averages over stochastic trajectories. The task of performing explicit calculations by this method can be accomplished making use of the Martin-Siggia-Rose-DeDominicis-Janssen formalism [5, 9-11]: in practice the procedure amounts to solve the problem of a Fokker-Planck operator which, for $\tau=\infty$, projects onto the stationary Euclidean weight in Eq. (7) (for details see [2]).

\section{Symplectic Quantization}

In order to proceed in the direction of an alternative approach to stochastic quantization, let us observe that the stochastic formalism of Eq. (9) allows one to introduce a key concept: the non-equilibrium relaxation dynamics for a relativistic quantum field, which asymptotically approaches an equilibrium value of the field correlation functions. The dynamical process of quantum fluctuations which brings the relativistic field across its phase-space with probability $\exp \left(-S^{(+)}[\phi] / \hbar\right)$ is clearly parametrized only by the intrinsic time $\tau$, while $x^{0}=c t$ can be formally considered as a standard coordinate.

In order to illustrate what we mean by non-equilibrium in the context of quantum field theory let us recall first the physical meaning of the equilibrium propagator:

$$
G\left(y^{0}, \mathbf{y} \mid x^{0}, \mathbf{x}\right)=\left\langle\phi\left(x^{0}, \mathbf{x}\right) \phi\left(y^{0}, \mathbf{y}\right)\right\rangle .
$$

The expression in Eq. (12) represents a measurement of how much a certain value of the field at coordinate time $x^{0}$ and position $\mathbf{x}$ is correlated to a value of the same field at another coordinate time $y^{0}>x^{0}$ and position $\mathbf{y}$. This correlation in space-time is usually evaluated assuming the equilibrium distribution $\exp \left(-S^{(+)}[\phi] / \hbar\right)$. But there might be special situations, e.g. relaxation from very unlikely configurations following the dynamics of Eq. (9), where, by repeating many times the measurements at 
time $x^{0}$ and position $\mathbf{x}$ and comparing them with the measurements of the same field at the following time $y^{0}$ and position $\mathbf{y}$ (it could have been even $\mathbf{y}=\mathbf{x}$ ), one may possibly find results not in agreement with the probability measure $\exp \left(-S^{(+)}[\phi] / \hbar\right)$. Assuming that the theory is correct, a possible explanation of such a behavior is that the field has not yet reached typical configurations with respect to the euclidean path integral measure. Of course this situation, where the field has not yet reached equilibrium, must be parametrized by another time variable, different from the coordinate time that we use to locate events in the space-time continuum. Such a variable cannot be anything else than the intrinsic time $\tau$ of stochastic quantization. Such a non-equilibrium regime would be described by the full time-dependent solution (usually out of reach) of the Fokker-Planck equation associated to Eq. (9). From the knowledge of this solution we would have a knowledge of the time-dependent propagator:

$$
G\left(y^{0}, \mathbf{y} ; \tau \mid x^{0}, \mathbf{x} ; \tau\right)=\left\langle\phi\left(y^{0}, \mathbf{y} ; \tau\right) \phi\left(x^{0}, \mathbf{x} ; \tau\right)\right\rangle .
$$

Coming back to what described in the previous section, one can interpret $\mathcal{L}^{(+)}\left(\phi, \partial_{\mu} \phi\right)$ in Eq. (5) as the potential part of the following Hamiltonian density (see also [12]):

$$
\begin{aligned}
\mathcal{H}_{E}(\phi, \pi) & =T-\mathcal{L}_{E} \\
& =\frac{1}{2} \frac{1}{c_{m}^{2}}\left(\frac{\partial \phi}{\partial \tau}\right)^{2}+\frac{1}{2} \sum_{\mu=0}^{3}\left(\frac{\partial \phi}{\partial x^{\mu}}\right)^{2}+V(\phi) \\
& =\frac{1}{2} \pi^{2}(x)+\mathcal{L}^{(+)}\left(\phi, \partial_{\mu} \phi\right),
\end{aligned}
$$

where the momentum

$$
\pi(x, \tau)=\frac{1}{c_{m}} \frac{\partial \phi}{\partial \tau}
$$

is canonically conjugated to $\phi(x, \tau)$ and the subscript $E$ in $\mathcal{H}_{E}(\phi, \pi)$ denotes the Euclidean version of our effective Hamiltonian. The constant $c_{m}$, which has to be made explicit for dimensional reasons, is a velocity. In this framework, it can be assumed as a free parameter of the theory. On the other hand, lacking in a relativistic invariant scalar field theory physical parameters other than $c$ and $\hbar$, there is a priori no reason for preventing the equivalence $c_{m}=c$, which therefore we will consider in what follows.

We can thus introduce the Euclidean action associated to the effective Hamiltonian density $\mathcal{H}_{E}(\phi)$ :

$$
\begin{aligned}
\mathcal{A}_{E}(\phi, \pi) & =\int d^{4} x \mathcal{H}_{E}(\phi, \pi) \\
& =S^{(+)}[\phi]+\frac{c^{2}}{2} \int d^{4} x \pi^{2}(x, \tau)
\end{aligned}
$$


In analogy with a classical Hamiltonian formulation, one can define the symplectic dynamics generated by the action $A_{E}$ as follows

$$
\begin{aligned}
& \frac{\partial \phi(x, \tau)}{\partial \tau}=\frac{\delta \mathcal{A}_{E}}{\delta \pi(x, \tau)}=\pi(x, \tau) \\
& \frac{\partial \pi(x, \tau)}{\partial \tau}=-\frac{\delta \mathcal{A}_{E}}{\delta \phi(x, \tau)}=-\frac{\delta S^{(+)}}{\delta \phi(x, \tau)}
\end{aligned}
$$

Thus, it turns out that the dynamics of quantum fluctuations in a relativistic field theory is ultimately ruled by a symplectic dynamics. Then, on the basis of the same assumption of "bona fide" ergodicity, which is usually taken for granted in most Hamiltonian systems, we can postulate the existence of a microcanonical ensemble for the fluctuations of the relativistic field $\phi(x)$. That is, we assume the following "microcanonical" postulate:

"All the configurations of the field $\phi(x)$ and the conjugated momentum $\pi(x)$ corresponding to an identical value of the action $\mathcal{A}_{E}(\phi, \pi)$ are attained with identical probability".

Accordingly, the equilibrium field theory can be obtained from a microcanonical statistical ensemble with partition volume:

$$
\Omega_{E}(A)=\int \mathcal{D} \phi \mathcal{D} \pi \delta\left(A-\mathcal{A}_{E}(\phi, \pi)\right),
$$

where $A$ denotes a given value of the action. The entropy, i.e. the generating functional of all connected diagrams of the relativistic field theory, reads

$$
\Sigma_{E}=k \log \Omega_{E}(A) .
$$

On the other hand, after having sketched above the construction of a relativistic invariant microcanonical approach to field-theory, one can realize that Wick's rotation, introduced for obtaining a statistical measure analogous to the Boltzmann-Gibbs canonical measure, is unnecessary in this context. Accordingly, nothing prevents us from making a step backward to the original field theory, equipped with its natural Minkowski metric, and considering the corresponding Hamiltonian density

$$
\begin{aligned}
\mathcal{H}_{M}(\phi, \pi) & =T-\mathcal{L} \\
& =\frac{c^{2}}{2} \pi^{2}(x, \tau)-\frac{1}{2}\left(\frac{\partial \phi}{\partial x^{0}}\right)^{2}+\frac{1}{2} \sum_{\mu=1}^{3}\left(\frac{\partial \phi}{\partial x^{i}}\right)^{2}+V(\phi)
\end{aligned}
$$

yielding the Minkowski generalized action

$$
\begin{aligned}
\mathcal{A}_{M}(\phi, \pi) & =\int d^{4} x \mathcal{H}_{M}(\phi, \pi) \\
& =\frac{c^{2}}{2} \int d^{4} x \pi^{2}(x, \tau)-S[\phi]
\end{aligned}
$$


which allows us to define the corresponding partition volume

$$
\Omega_{M}(A)=\int \mathcal{D} \phi \mathcal{D} \pi \delta\left(A-\mathcal{A}_{M}(\phi, \pi)\right)
$$

with the entropy

$$
\Sigma_{M}=k \log \left[\Omega_{M}(A)\right] .
$$

Analogously, the symplectic dynamics (17) changes to

$$
\begin{aligned}
& \frac{\partial \phi(x, \tau)}{\partial \tau}=\frac{\delta \mathcal{A}_{M}}{\delta \pi(x, \tau)}=\pi(x, \tau) \\
& \frac{\partial \pi(x, \tau)}{\partial \tau}=-\frac{\delta \mathcal{A}_{M}}{\delta \phi(x, \tau)}
\end{aligned}
$$

Some important remarks are necessary. First of all, we have to point out that, at variance with $\mathcal{A}_{E}$, the Minkowski action $\mathcal{A}_{M}$ is not a definite sign quantity, as one can immediately infer from (20) and (21). Accordingly, in principle one is not allowed to follow the formal classical pathway to recover a canonical partition function $\mathcal{Z}(\beta)$ by Laplace-transforming $\Omega_{M}(A)$ :

$$
\mathcal{Z}(\beta)=\int_{0}^{\infty} d A e^{-\beta A} \Omega_{M}(A) .
$$

The Laplace transform of Eq. (25) is well defined, for either positive or negative values of $\beta$, only when the variable $A$ has a definite sign, which is not the case for a relativistic field theory. As a consequence, the only transform that one can consistently define in complete generality (i.e., not limited to a specific interval of values of $A)$ is the Fourier-transform $\mathcal{Z}(z)$ :

$$
\begin{aligned}
\mathcal{Z}(z) & =\frac{1}{\sqrt{2 \pi}} \int_{-\infty}^{\infty} d A e^{-i z A} \Omega_{M}(A) \\
& =\int \mathcal{D} \phi \mathcal{D} \pi e^{-i z \mathcal{A}_{M}(\pi, \phi)} .
\end{aligned}
$$

It is for this reason that the building blocks of quantum field theory are probability amplitudes rather than probabilities. Due to the separable form of $\mathcal{A}_{M}(\pi, \phi)$, the integration over momenta can be straightforwardly performed because of its quadratic dependence on $\pi(x)$ and the result can be hidden inside the infinite normalization constant $\mathcal{N}$, typical of field theories. Now, if we fix $z=1 / \hbar$, we recover the standard form of the Feynman path integral:

$$
\begin{aligned}
\mathcal{Z}\left(\hbar^{-1}\right) & =\int \mathcal{D} \phi \mathcal{D} \pi e^{\frac{i}{\hbar} S[\phi]-\frac{i}{\hbar} \frac{c^{2}}{2} \int d^{4} x \pi^{2}(x, \tau)} \\
& =\mathcal{N}(\hbar) \int \mathcal{D} \phi e^{\frac{i}{\hbar} S[\phi]}
\end{aligned}
$$


The role played by $\hbar$ is analogous, with respect to the conservation of the generalized action in Eq. (21), to the one played by temperature in the canonical ensemble of statistical mechanics with respect to the conservation of energy. Accordingly, the role of the canonical partition function is played by the Feynman path-integral, while the role of the microcanonical partition sum is played by the expression in Eq. (22). Let us point out that the microcanonical partition function in Eq. (22) is not merely an equivalent way to write the Feynman path integral, but it has a completely new probabilistic interpretation as a field-theoretic functional integral. This is particularly clear if we put the theory on a lattice: while the Feynman path integral still lacks an interpretation in terms of probability, unless we consider the corresponding Euclidean field theory by rotating time to the imaginary axis, the microcanonical partition function $\Omega_{M}(A)$ yields immediately a probability for the field configurations while keeping $\mathcal{A}_{M}[\phi, \pi]$ with its original Lorentzian metric:

$$
P_{A}[\phi, \pi]=\frac{1}{\Omega_{M}(A)} \delta\left(A-\mathcal{A}_{M}[\phi, \pi]\right) .
$$

Just to mention one possible application, the symplectic quantization approach allows one to evaluate by means of lattice field theory techniques the "equilibrium" (with respect to the above mentioned symplectic dynamics) correlations between light-cone events, typically occurring in the study of parton distribution functions [13], which are typically unaccessible in Euclidean field theory. Moreover, one has the possibility of studying the physical dynamics of quantum fluctuations by means of Eq. (24), making use, for instance, of a lattice version of the theory. Note that an approach allowing one to study the relaxation of quantum fluctuations in relativistic fields by means of a deterministic dynamics might be particularly relevant in the context of inflation theory [14-17]. In this perspective it is worth mentioning the recent results on the stochastic quantization approach to the gravitational field discussed in [18]: according to these results, the whole era before the end of inflation is characterized by the impossibility to rotate coordinate time from immaginary back to the real axis, so that the only variable which governs the dynamics is the fictitious time of stochastic quantization, corresponding to our intrinsic time.

It thus happens that, while in statistical mechanics ensembles at fixed temperature or fixed energy are usually equivalent, this is not apparently true for quantum field theories, with action taking the place of energy. In quantum field theory it seems that the "pseudo-microcanonical fixed-action ensemble" introduced here [Eq. (22)] provides a more robust description of the system, in particular it looks like that the path-integral formulation is more severly plagued by the appearance of divergences with respect to symplectic quantization. The reason is the following: while the renormalization procedure in quantum field theories results in the existence of an additional action scale to be fixed by hand (the invariant energy scale at which the predictions of the theory are compared with experiments) [3, 5], symplectic quantization fixes such a scale from the very beginning. Moreover, in the context of symplectic quatization, functional integrals such as the partition function in Eq. (22) are well defined irrespectively to the renormalizability of the theory. 
In summary, the path-integral formulation of quantum field theory can be viewed as a representation dual to symplectic quantization. Within the path-integral the role of quantum fluctuations is assumed to be reduced to a natural scale factor, $\hbar$, thus epitomizing the role of the dynamical degrees of freedom $\pi(x)$ into a normalization constant. The overall procedure taking from symplectic quantization to the Feynman path integral looks similar to the ergodicity assumption in the Boltzmann theory of an ideal gas. But there is an important difference: in the case of symplectic quantization the dynamical degrees of freedom $\pi(x)$ really seem to play the role of the hidden variables invoked by Einstein to allow for a complete causal description of quantum phenomena.

\section{Conclusions and Perspectives}

The approach illustrated in this manuscript aims at recasting the relations among relativistic field theory, quantum fluctuations and statistical mechanics. By assuming the agnostic point of view according to which it is not assumed the existence of elementary degrees of freedom for matter (although our theory might be consistent with this hypothesis) we have proposed an approach to relativistic fields based on a statistical ensemble characterized by a fixed value of the action, rather than of the energy. Our approach was inspired by Parisi-Wu stochastic quantization [2], where the dynamics of quantum fluctuations for a relativistic field is parametrized by a fictitious time.

We have discussed how the Parisi-Wu stochastic quantization procedure is based on an extension of the statistical mechanics ensemble theory, through the strict analogy between the canonical partition function and the path-integral formalism, where the role of the temperature is taken by Planck's constant and the one of the Hamiltonian by the action of the field theory. On the other hand, when dealing with a relativistic field-theory the original action (the one defined in Minkowski space) is not positive definite and this implies that it would be much more natural to construct a statistical mechanics approach exploiting the analogy with a microcanonical equilibrium ensemble, where the value of the total action fixes the physical scale of the system, without allowing this quantity to fluctuate over all its possible values as it happens in a canonical framework. The most relevant outcome of our analysis is that, once we assume a microcanonical description, the fictitious time introduced by Parisi and $\mathrm{Wu}$ [2] can be regarded as a true physical entity: it is the parameter which rules the evolution of quantum fluctuations and allows us to consistently extend to quantum field theory concepts as non-equilibrium dynamics, relaxation to equilibrium and irreversibility. Since this variable is related to the "internal" dynamics of quantum fluctuations at each point of space-time and its definition comes irrespectively to any choice of reference frame we called it intrinsic time. Of course, coordinate time is still there, but, as the name says, it simply plays the role of a coordinate to locate events. Clearly, time intervals along the relaxational dynamics parametrized by the intrinsic time has to be measured in terms of the coordinate time. In order to dispel any doubt about this point, we want to stress that symplectic quantization does not imply the existence of any universal time-scale for dynamics. 
In other words, symplectic quantization is not associated to the existence of a universal clock, which would violate the principles of relativity, for the simple reason that the characteristic time-scale for the dynamics of $\phi(x, \tau)$ can fluctuate across different regions of the space-time continuum. In addition, let us point out that the elapsing of intrinsic time can be measured only indirectly, for instance looking at the irreversible relaxation from very atypical configurations of the fields to typical ones. A problem of this kind is the one studied in [20] in the context of inflationary cosmology.

The notion of intrinsic time that we propose here is quite close to that of thermal time proposed in [19]. We have simply highlighted that, in the case of a relativistic field, a definition of time conceptually analogous to that of [19] is perfectly consistent for a relativistic system without any need to consider a finite temperature.

As a test of the new symplectic quantization formalism ideas, we have applied them to the study of the gravitational field quantum fluctuations in [1], a work where the presence of a cosmological constant term in Einstein equations is put in relation with the existence of "kinetic" degrees of freedom (in the sense of intrinsic time) for the metric field.

Acknowledgements We warmly thank for useful discussions S. Caracciolo, P. Di Cintio, S. Matarrese, A. Riotto and A. Vulpiani. In particular we thank S. Matarrese for a careful reading of the manuscript. G.G. acknowledges Sapienza university of Rome, Physics Department, for kind hospitality during some periods in the preparation of this manuscript. R.L. acknowledges partial support from project MIURPRIN2017 Coarse-grained description for non-equilibrium systems and transport phenomena (CONEST) No. 201798CZL.

Funding Open access funding provided by Gran Sasso Science Institute - GSSI within the CRUI-CARE Agreement.

Open Access This article is licensed under a Creative Commons Attribution 4.0 International License, which permits use, sharing, adaptation, distribution and reproduction in any medium or format, as long as you give appropriate credit to the original author(s) and the source, provide a link to the Creative Commons licence, and indicate if changes were made. The images or other third party material in this article are included in the article's Creative Commons licence, unless indicated otherwise in a credit line to the material. If material is not included in the article's Creative Commons licence and your intended use is not permitted by statutory regulation or exceeds the permitted use, you will need to obtain permission directly from the copyright holder. To view a copy of this licence, visit http://creativecommons.org/licen ses/by/4.0/.

\section{References}

1. Gradenigo, G.: Symplectic quantization II: dynamics of space-time quantum fluctuations and the cosmological constant. arxiv:2101.01795 (2021)

2. Parisi, G., Wu, Y.: Perturbation theory without gauge fixing. Sci. Sin. 24, 483-496 (1981)

3. Ramond, P.: Field theory: a modern primer. Frontiers in Physics. Benjamin/Cummings Publishing Co., San Francisco (1981)

4. Daamgard, P.H., Hüffel, H.: Stochastic Quantization. World Scientific, Singapore (1988)

5. Zinn-Justin, J.: Quantum Field Theory and Critical Phenomena. Oxford Science Publications, Oxford (1989) 
6. Gradenigo, G., Iubini, S., Livi, R., Majumdar, S.N.: Localization transition in the discrete non-linear Schrödinger equation: ensembles inequivalence and negative temperatures. J. Stat. Mech. (2021). https://doi.org/10.1088/1742-5468/abda26

7. Ryang, S., Saito, T., Shigemoto, K.: Canonical stochastic quantization. Prog. Theor. Phys. Lett. 73, 1295-1298 (1985)

8. Rumpf, H.: Stochastic quantization of Einstein gravity. Phys. Rev. D 33, 942 (1986)

9. Martin, P.C., Siggia, E.D., Rose, H.A.: Statistical dynamics of classical systems. Phys. Rev. A 8, 423 (1973)

10. Janssen, H.-K.: On a Lagrangean for classical field dynamics and renormalization group calculations of dynamical critical properties. Zeit. für Phys. B: Cond. Mat. 23, 377 (1976)

11. De Dominicis, C.: Dynamics as a substitute for replicas in systems with quenched random impurities. Phys. Rev. B 18, 4913 (1978)

12. Ryang, S., Saito, T., Shigemoto, K.: Canonical stochastic quantization. Prog. Theor. Phys. 73, 5 (1985)

13. Ji, X.: Parton physics on a Euclidean lattice. Phys. Rev. Lett. 110, 262002 (2013)

14. Mollerach, S., Matarrese, S., Ortolan, A., Lucchin, F.: Stochastic inflation in a simple two-fiel model. Phys. Rev. D 44, 1670 (1994)

15. Gangui, A., Lucchin, F., Matarrese, S., Mollerach, S.: The three-point correlation function of the cosmic microwave background in inflationary models. Astrophys. J. 430, 447-457 (1994)

16. Pinol, L., Renaux-Petel, S., Tada, Y.: A manifestly covariant theory of multifield stochastic inflation in phase space. arXiv:2008.07497 (2020)

17. Pattison, C., Vennin, V., Wands, D., Assadullahi, H.: Ultra-slow-roll inflation with quantum diffusion.arXiv:2101.05741 (2021)

18. Baulieu, L., Wu, S.: Second Order Langevin Equation and Definition of Quantum Gravity By Stochastic Quantisation. arXiv:1807.11255 (2020)

19. Connes, A., Rovelli, C.: Von Neumann algebra automorphisms and time-thermodynamics relation in generally covariant quantum theories. Class. Quantum Grav. 11, 2899 (1994)

20. Di Cintio, P., Gradenigo, G., Livi, R.. Riotto, A.: Symplectic quantization and the slow dynamics of reheating: formation and decay of cosmological breathers (in preparation)

Publisher's Note Springer Nature remains neutral with regard to jurisdictional claims in published maps and institutional affiliations. 